\title{
Karen Pratt, La Mort le roi Artu
}

\section{Richard Trachsler}

\section{(2) OpenEdition}

\section{Journals}

\section{Édition électronique}

URL : http://journals.openedition.org/studifrancesi/29953

DOI : 10.4000/studifrancesi.29953

ISSN : 2427-5856

\section{Éditeur}

Rosenberg \& Sellier

\section{Édition imprimée}

Date de publication : 1 avril 2006

Pagination : 128-129

ISSN : 0039-2944

\section{Référence électronique}

Richard Trachsler, "Karen Pratt, La Mort le roi Artu », Studi Francesi [En ligne], 148 (XLX | I) | 2006, mis en ligne le 30 novembre 2015, consulté le 21 avril 2021. URL : http://journals.openedition.org/ studifrancesi/29953; DOI : https://doi.org/10.4000/studifrancesi.29953

\section{Ce document a été généré automatiquement le 21 avril 2021.}

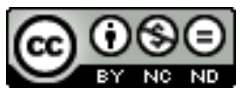

Studi Francesi è distribuita con Licenza Creative Commons Attribuzione - Non commerciale - Non opere derivate 4.0 Internazionale. 


\title{
Karen Pratt, La Mort le roi Artu
}

\author{
Richard Trachsler
}

\section{RÉFÉRENCE}

KAREN PRATT, La Mort le roi Artu, London, Grant \& Cutler Ltd. («Critical Guides to French Texts », 137), 2004, pp. 112 .

1 Ce svelte livre bleu de petit format est le $137^{\mathrm{e}}$ de la collection bien connue des Critical Guides to French Texts, où les œuvres médiévales sont assez régulièrement à l'honneur : après Chrétien de Troyes, Marie de France, Villon, les textes tristaniens, le Roman de la Rose et quelques grands textes épiques, c'est maintenant au tour de la Mort Artu d'accéder au rang de «classique » de la littérature française. On comprend aisément ce choix des directeurs de la collection, puisque la dernière partie du Lancelot-Graal, qui relate l'implosion du royaume arthurien, exerce depuis longtemps sur les lecteurs une fascination immédiate. C'est même certainement la partie du cycle qui se rapproche le plus de l'idée qu'un lecteur non spécialiste se fait d'un roman tout court, mais c'est aussi un texte qui reprend, à l'instar des autres volets du Lancelot-Graal, toute une tradition littéraire existante par rapport à laquelle se déploie son sens. C'était donc un des défis auxquels devait répondre Karen Pratt : mettre à nu les rouages d'une écriture en apparence sans artifices qui semble se comprendre toute seule, alors qu'il s'agit, en réalité, d'une œuvre élaborée et complexe, qui se nourrit de nombreux avant-textes. En d'autres termes, elle devait montrer la construction de l'œuvre sans la disséquer et lui ôter le charme qui, à coup sûr, opère sur un public estudiantin de bonne foi.

2 Conformément aux objectifs de la collection, destinée aux étudiants de français dans les universités anglophones, le guide ne présuppose rien en matière de lectures médiévales. Sans pédanterie, mais sans trop s'attarder non plus, Karen Pratt fournit donc toutes les informations nécessaires pour entamer la lecture de la Mort Artu de façon adéquate. Dans une première partie (Rewriting Arthurian Tradition : the context, $\mathrm{p}$. 9-25), elle rappelle que le livre gris qui abrite le texte de l'édition Frappier n'existait pas au Moyen Age et que la Mort Artu est transmise - toujours - dans le cadre d'une partie 
du cycle du Lancelot-Graal. Elle présente donc ce cycle, évoque Gautier Map, l'architecte de Frappier, Geoffroy de Monmouth et Wace, Chrétien de Troyes, le Graal et bien d'autres points encore en une quinzaine de pages. Quand il y a controverse à propos d'un aspect - et rares sont les questions qui ont toujours fait l'unanimité - Karen Pratt le signale brièvement, ce qui permet à un étudiant de comprendre ce qui lui arrive quand il tombe, par hasard, sur une étude critique qui ne reflète pas ce qu'il vient d'apprendre. Miraculeusement, on a l'impression que ce premier chapitre, difficile à ne pas rater, reste compréhensible pour un néophyte. Le spécialiste, en tout cas, ne trouvera rien à y redire : c'est sans surcharge, solide et parfaitement to the point. A la $\mathrm{p}$. 26, le lecteur est donc au seuil de la Mort Artu même, il la découvrira à travers quatre parties, qui, ensemble, proposent un parcours de lecture classique, mais efficace :2. The Tragedy of Arthur's Death: the characters and the plot (p. 26-46), 3. Interpreting Arthurian History: the quest for meaning (47-66), 4. Arthurian Values: condemnation or commemoration? (p. 67-85), 5. The Art of the Prose Romancer (p. 86-101). L'idée de commencer par la présentation des personnages et de l'intrigue, donc en "clé dramatique", était excellente du point de vue pédagogique. Non seulement, on découvre ainsi les protagonistes les plus importants et leurs actions, mais on a aussi l'occasion de penser, comme l'avait exposé Karen Pratt dans une étude antérieure, le texte comme une tragédie, sur fond boécien ou augustinien, et de poser donc un grand nombre de questions qui informeront les autres parties. Qui tire les ficelles? Dieu? Fortune ? Y a-t-il eu faute? d'Arthur? de Lancelot? Les instances politiques, tiraillées entre les différents clans, sont-elles critiquées ou s'agit-il de dysfonctionnements dus aux hommes? Les valeurs arthuriennes sont-elles supplantées par des valeurs plus spirituelles? L'amour est-il un atout ou un frein au bonheur? En soulevant les grands problèmes de ce type, Karen Pratt pose les questions qui permettent de s'interroger sur le texte. Elle le fait très simplement, tout jargon est banni de sa prose, de même qu'elle n'assène aucune vérité préconçue. Les réponses, siennes ou d'autrui, sont plutôt esquissées, mais elle n'hésite pas à prendre position contre les grandes figures de la médiévistique de naguère (Frappier) ou d'aujourd'hui (Bloch) quand leurs arguments ne lui semblent pas corroborés par le texte. Au final, aucune thèse choc n'émerge de la lecture de l'étude de Karen Pratt, mais quelques acquis simples: la Mort Artu est une histoire assez universelle qui parle d'amour et de pouvoir, mais ancrée dans le milieu social du XIII ${ }^{e}$ siècle qui lit à sa façon ce roman de chevalerie. C'est aussi un roman qui met en scène des personnages sans cesse confrontés à des signes qu'ils doivent interpréter. Jusqu'à un certain point, c'est donc aussi un roman qui demande au lecteur de fournir ce même effort, d'autant plus qu'aucune instance narrative n'intervient pour l'accomplir à sa place. Cette dernière mise en abyme est la seule concession aux canons de l'exégèse moderne de la part de Karen Pratt. Mais l'idée est d'autant plus défendable que cette équivalence entre lecteur moderne et personnage médiéval n'est pas posée d'emblée comme axe de lecture, mais apparait plutôt comme un résultat en cours de la réflexion.

3 L'étudiant n'aura aucune difficulté à suivre le raisonnement du livre, mais seul le lecteur expérimenté saura que, sous ses dehors un peu sages, se cache un travail longuement mûri et bien plus profond, sans doute, que la limpidité de la formulation et de l'argumentation ne laissent penser. Le tout est équilibré, évitant excès et surinterprétations. Le livre échappe ainsi à tout effet de mode, et je crois qu'il vieillira bien. 
4 Notre collègue londonienne a donc pleinement rempli son contrat. Elle a réalisé un guide lisible et parfaitement informé, qui donne aux lecteurs toutes les clés nécessaires pour entrer dans la dernière partie du grand édifice du Lancelot-Graal afin de continuer l'exploration par eux-mêmes. C'est cela même, l'idée d'un bon guide : rendre attentifs les visiteurs à ce qu'il y a à voir, au lieu de leur prescrire un itinéraire et leur imposer une exégèse finie. Aux étudiants, on recommandera donc sans hésitation ce Critical Guide, pour les collègues, c'est une bonne occasion de reprendre le texte de la Mort Artu et de vérifier ce qu'en dit Karen Pratt (p. 28): Aristotle was right that family relationships make the best tragedy. 